\title{
How to Predict the Long-term Course of Neurodegenerative Diseases?
}

\author{
Alberto Montolío ${ }^{1}$, José Cegoñino ${ }^{1}$, Elena Garcia-Martin ${ }^{2}$, Amaya Pérez del Palomar ${ }^{1}$ \\ ${ }^{1}$ Group of Biomaterials (GBM) \\ Aragon Institute of Engineering Research (I3A) \\ University of Zaragoza, Mariano Esquillor s/n, 50018, Zaragoza, Spain. \\ Tel. +34-976762707, e-mail:amontolio@unizar.es \\ ${ }^{2}$ Ophthalmology Department, Miguel Server University Hospital, Zaragoza, Spain
}

\begin{abstract}
The aim of this work is to predict the disability state in neurodegenerative disease, such as multiple sclerosis (MS), using clinical batabases and machine learning techniques. This prediction could help clinicians select a more specific treatment for MS patients.
\end{abstract}

\section{Introduction}

Some of the most devastating neurodegenerative diseases induce axonal degeneration in the central nervous system, including the optic nerve. Axonal loss is considered the main cause of functional disability in MS, but the detection of axonal injury has typically been difficult. Several studies carried out to date [1],[2] have shown that there is a greater thinning of retinal nerve fiber layer (RNFL) in patients affected by MS than in healthy controls. This fact has established RNFL as a MS biomarker.

There are several non-invasive imaging techniques to measure the thickness of different layers of retina, but the most widespread technique is optical coherence tomography (OCT) since it is cost-effective, rapid and reproducible. Due to OCT allows to measure specifically the thickness of RNFL and even the ganglion cell layer, this method is used to diagnose and monitor multiple diseases of retina and optic nerve.

Machine learning techniques can be used to improve the diagnosis and predict the evolution of some complex diseases. In this field, Garcia-Martin et al. used neuronal networks to diagnose MS using OCT [3]. Pérez del Palomar et al. demonstrated that machine learning is a useful tool to detect MS disease [4]. The next step is to apply these techniques to predict the course of the disease, the result may be useful for clinicians to decide treatment in each patient. The work performed by Zhao et al. [5] managed to predict the evolution of disability state in short-term course using support vector machines.
Other computational classifiers, such as decision tree, linear tree and linear regression, was tested to predict MS evolution within two years [6]. However, OCT data and machine learning have not been used to predict the long-term course of MS disease.

In this study, machine learning techniques were used to evaluate the ability of clinical data and OCT measurements to predict the disability state in MS patients.

\section{Materials and methods}

A longitudinal study from Miguel Servet Hospital with 82 MS patients was used, the 10-year follow-up was carried out by ophthalmologists to perform OCT measurements and by neurologists to determine the expanded disability status scale (EDSS) value. One eye from each white European origin subject was randomly selected, excluding eyes with previous episodes of optic neuritis.

The input data of our predictive model were: age, sex, best-corrected visual acuity (BCVA), MS duration, MS subtype, optic neuritis antecedent, relapse in preceding year, EDSS value and the 6 RNFL thicknesses. And the output was "worsening" or "non-worsening" in the long-term EDSS value. "Worsening" was defined as an increase of 1 or more points in EDSS and "non-worsening" represented MS patients whose EDSS increased less than 1 point or decreased. Least absolute shrinkage and selection operator (LASSO) was used to remove irrelevant features and reduce the risk of overfitting. After the implementation of this algorithm, 5 features were chosen: MS duration, relapse in preceding year, EDSS score, temporal RNFL thickness and superior RNFL thickness. Our predictive model used these 5 features collected at the baseline visit and the first two annual follow-up visits to predict the disability state of MS patients 8 years later. Several classification algorithms were tested to evaluate this predictive model. 


\section{Results}

Long short-term memory (LSTM) recurrent neural network was the most appropriate classifier to predict whether or not the disability state of MS patients would worsen. The prediction was correct in 67 of 82 MS patients ( 8 false positves and 7 false negatives, see confusion matrix in Figure 1), giving an accuracy of $81.7 \%$. The area under curve (AUC) was 0.8165 . Therefore, this result underlines the potential of RNFL thickness as a MS biomarker.

\section{Conclusion}

Since in this neurodegenerative disease the disability appears at an early stage of the disease, it is very important to make an early diagnosis and predict the disease evolution with the aim of applying more specific treatments to stop the disability progress. OCT is an objective and reproducible test that can be perfomed in just two minutes by non-specialized personal.

As can be seen in this work, machine learning is able to use clinical databases to improve diagnosis and predict the progress in MS. Our study demonstrates that these techniques, using clinical data and RNFL thickness, turn out to have a great ability to classify new patients according to their MS progression.

\section{Acknowledgements}

This work was supported by the Spanish Ministry of Economy and Competitiveness through project DPI 2016-79302-R, by the Spanish Ministry of Science,
Innovation and Universities under grant BES-2017080384 and by PI17/01726 (Instituto de Salud Carlos III).

\section{References}

[1]. GRAZIOLI, E., ZIVADINOV, R., WEINSTOCKGUTTMAN, B., et al. Retinal nerve fiber layer thickness is associated with brain MRI outcomes in multiple sclerosis. Journal of the Neurological Sciences. 2008, 268(1-2), 12-17.

[2]. GARCIA-MARTIN, E., POLO, V., LARROSA, JM., et al. Retinal Layer Segmentation in Patients with Multiple Sclerosis Using Spectral Domain Optical Coherence Tomography. Ophthalmology. 2014, 121(2),573-579.

[3]. GARCIA-MARTIN, E., PABLO, LE., HERRERO, R., et al. Neural networks to identify multiple sclerosis with optical coherence tomography. Acta Ophthalmologica. 2013, 91(8), e628-e634.

[4]. PEREZ DEL PALOMAR, A., CEGOÑINO, J., MONTOLIO, A., et al. Swept source optical coherence tomography to early detect multiple sclerosis disease. The use of machine learning techniques. PLoS One. 2019, 14(5), e0216410.

[5]. ZHAO, Y., HEALY, BC., ROTSTEIN, D., et al. Exploration of machine learning techniques in predicting multiple sclerosis disease course. PLoS One. 2017, 12(4), e0174866.

[6]. BEJARANO, B., BIANCO, M., GONZALEZMORON, D., et al. Computational classifiers for predicting the short-term course of Multiple sclerosis. BMC Neurology. 2011, 11(1), 67.
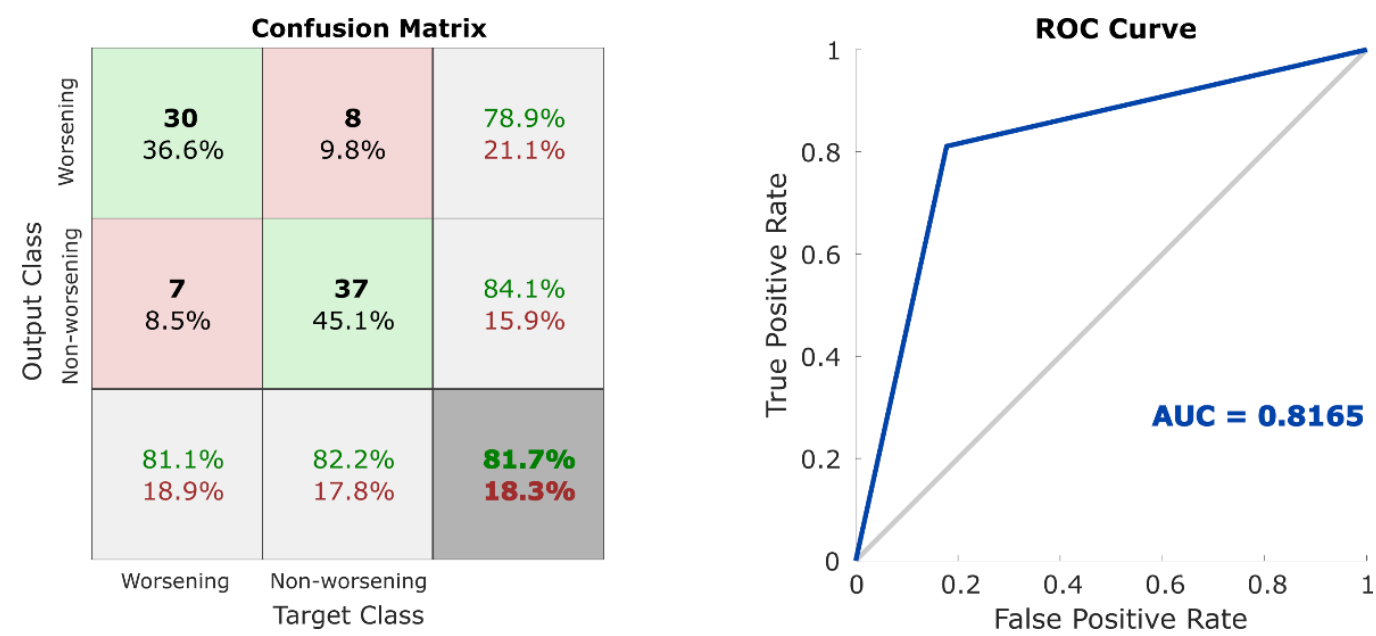

Figure 1. Confusion matrix and receiver operating characteristic (ROC) curve, with area under curve (AUC), using the long short-term memory (LSTM) recurrent neural network to predict the disability state in patients with multiple sclerosis. 